\title{
Si and $\mathrm{Si}_{x} \mathrm{Ge}_{1-x} \mathrm{NWs}$ studied by Raman spectroscopy
}

\author{
J. Anaya ${ }^{1}$, A. C. Prieto ${ }^{1}$, O. Martínez ${ }^{1 *}$, A. Torres ${ }^{1}$, A. Martín-Martín ${ }^{1}$, J. Jiménez $^{1}$, A. Rodríguez ${ }^{2}$, \\ J. Sangrador ${ }^{2}$, and T. Rodríguez ${ }^{2}$ \\ ${ }^{1}$ GdS Optronlab, Dpto. Física de la Materia Condensada, Edificio I+D, Paseo de Belén 1, 47011 Valladolid, Spain \\ ${ }^{2}$ Dpto. Tecnología Electrónica, E. T. S. I. de Telecomunicación, Universidad Politécnica de Madrid, 28040 Madrid, Spain
}

Keywords Si, nanowires, Raman spectroscopy, phonon confinement model, heat dissipation, finite element analysis

\footnotetext{
* Corresponding author: e-mail oscar@fmc.uva.es, Phone/Fax: +34 983184956
}

Group IV nanostructures have attracted a great deal of attention because of their potential applications in optoelectronics and nanodevices. Raman spectroscopy has been extensively used to characterize nanostructures since it provides non destructive information about their size, by the adequate modeling of the phonon confinement effect. The Raman spectrum is also sensitive to other factors, as stress and temperature, which can mix with the size effects borrowing the interpretation of the
Raman spectrum. We present herein an analysis of the Raman spectra obtained for $\mathrm{Si}$ and $\mathrm{SiGe}$ nanowires; the influence of the excitation conditions and the heat dissipation media are discussed in order to optimize the experimental conditions for reliable spectra acquisition and interpretation.
1 Introduction Nanosized structures of group IV semiconductors have been the object of many studies in the last years due to their potential applications [1-3]. Their properties greatly depend on their dimensions; also, the composition is crucial in the case of alloyed SiGe NWs. Raman spectroscopy is a very powerful non destructive tool suitable to such study, because the Raman spectrum is sensitive to both, composition, in alloyed systems, and dimensions, through the phonon confinement effect in reduced size structures [1-4]. However, both effects can mix and the interpretation of the Raman data is not an easy task. Moreover, other factors affect the Raman spectra, such as temperature and stress [5]. This could be beneficial, since a Raman study can also provide information about the thermal properties, such as the thermal expansion and the anharmonicity, which are crucial to model the heat dissipation and the thermal induced stresses, necessary to design devices with optimal mechanical and heat transport properties. The one phonon Raman spectrum of $\mathrm{Si}$ nanowires (NWs) has reached a great deal of controversy, because of the discrepancies between the expected and measured lineshapes, failing in the accurate estimation of the NW diame- ter [1-3]. Different reasons have been argued as possible causes for the inconsistency of the Raman parameters measured in Si NWs. The one phonon Raman lineshape can be modified by inhomogeneous NW diameter distribution [1], Fano scattering from photogenerated carriers [2], and laser induced NW heating [3]. In fact, one has to consider that the Raman microprobe works with a relative high laser power density; the energy absorbed by the sample needs to be dissipated. If the heat evacuation is not efficiently achieved the scattering volume can reach temperatures above the ambient, which deform the Raman bands in a non negligible amount. Therefore, the thermal dissipation of the probed system has to be considered if one looks for a straightforward interpretation of the Raman data. Usually, nanostructures are embedded in an environment medium, which can have poor thermal conductivity, as is the case of NWs in air. This, together with the very small base of the NW in contact with the substrate where they were grown, make difficult the heat dissipation from the NW; therefore, one can expect a non-negligible temperature enhancement in the NWs under the laser exposure during the Raman measurement. The temperature enhancement should de- 
pend on the surrounding media, the dimensions of the NWs, and the laser excitation conditions; e.g. wavelength and the incidence respect to the NW.

We present herein an analysis of the Raman spectra of $\mathrm{Si}$ and alloyed $\mathrm{Si}_{x} \mathrm{Ge}_{1-x} \mathrm{NWs}$, revealing the influence of the laser induced heating on the Raman spectrum. The temperature distribution inside the NWs is modelled using a finite element analysis procedure. Since the Raman signal is very low for a single $\mathrm{NW}$, due to the very small volume probed, the spectrum is generally acquired for bundles of NWs. This would provide averaged information over several NWs, which can have different diameters and lengths, and therefore can have very different responses to the laser excitation. Therefore, the analysis of individual NWs is necessary for the understanding of the one phonon Raman spectrum of these structures. An experimental protocol to prepare isolated NWs for microRaman observation was designed, consisting in separation of the NWs by ultrasonication and suspension in methanol, then being deposited on a lacey carbon coated copper grid holder as those used for TEM observations. The NWs were then identified by SEM images.

2 Experimental set-up $\mathrm{Si}$ and $\mathrm{Si}_{x} \mathrm{Ge}_{1-x} \mathrm{NWs}$ were fabricated by Low Pressure Chemical Vapour Deposition (LPCVD) on (100) $\mathrm{Si}$ substrates using $\mathrm{Si}_{2} \mathrm{H}_{6}$ and $\mathrm{GeH}_{4}$ as precursor gases. The Si substrates were dipped in HF, rinsed for $30 \mathrm{~s}$ in APTES, dried with $\mathrm{N}_{2}$ and subsequently rinsed in a colloidal suspension of Au particles $(30 \mathrm{~nm}$ in diameter) for $180 \mathrm{~s}$. The samples were immediately loaded into the LPCVD reactor, where they were annealed at 500 ${ }^{\circ} \mathrm{C}$ for $60 \mathrm{~min}$. The subsequent $\mathrm{NW}$ growth process was carried out also at $500{ }^{\circ} \mathrm{C}$ for $30 \mathrm{~min}$, using $\mathrm{GeH}_{4}$ flows between 2 and $20 \mathrm{sccm}$ and $\mathrm{Si}_{2} \mathrm{H}_{6}$ flows between 8 and 2 sccm, in such a way that the flow ratio $\mathrm{GeH}_{4}: \mathrm{Si}_{2} \mathrm{H}_{6}$ was varied between 0.25 and 10 . The total pressure was maintained at 400 mTorr.

The Raman spectra were acquired with a Labram UVHR 800 Raman spectrometer from Jobin Yvon. The excitation was done with an $\mathrm{Ar}^{+}$laser $(514.5 \mathrm{~nm})$ or a $\mathrm{He}-\mathrm{Ne}$ laser $(632.8 \mathrm{~nm})$, using a 0.95 numerical aperture $100 \mathrm{X}$ objective; excitation power densities on the sample surface up to $100 \mathrm{kWcm}^{-2}$ were used.

3 Results In the case of Si NWs, where no composition effects should be considered, the Raman spectrum of bundles of NWs, Fig. 1, has been interpreted according to the effects of the three aforementioned factors, e.g., the phonon confinement effect, the laser induced heating of the NWs and the dispersion in diameters. In fact, as observed in the SEM image of the NWs (inset in Fig. 1), they form tangles of wires, with diameters ranging from 15 to $120 \mathrm{~nm}$ and several $\mu \mathrm{m}$ length. The laser spot focused onto the sample surface has a diameter of $\sim 1 \mu \mathrm{m}$ in our experimental conditions, which implies that the Raman spectrum is giving simultaneous information of a reduced number of NWs, whose diameters can differ each other.

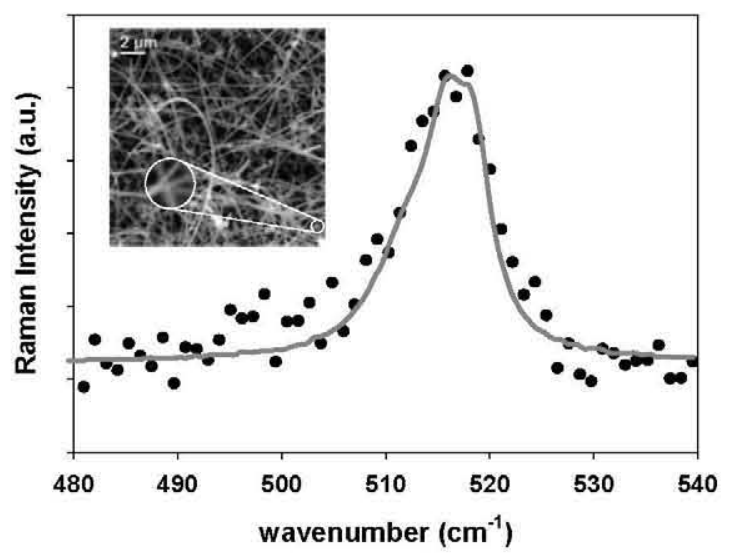

Figure 1 Raman spectrum of bundled Si NWs. Points: experimental data; line: calculated spectrum (see text). The inset shows a SEM image of the NWs, the circle indicates the diameter $(D)$ of the laser beam focused on the sample.

The effect of the reduced size of the NWs and the increase of the temperature due to the laser heating have been considered by means of a phenomenological phonon confinement model [6]. These models are based on the uncertainty of the phonon momentum, because of the reduced size of the structures. In reduced size structures phonons with $q \neq 0$ are allowed to contribute to the Raman spectrum. Assuming a Gaussian localization factor [6] the Raman intensity can be expressed as:

$$
I(\omega, L) \propto \int \exp \left(-\frac{q^{2} \phi^{2}}{16 \pi}\right) \frac{d^{3} q}{[\omega-\omega(q)]^{2}+\left(\frac{\Gamma_{O}}{2}\right)^{2}}
$$

where $\omega(q)$ is the phonon dispersion function and $\Gamma_{o}$ is the full width at half maximum (FWHM) of the bulk material; the linewidth includes the instrument broadening contribution, which in our experimental conditions gives a full width at half maximum for a high quality Si bulk crystal of $2.8 \mathrm{~cm}^{-1}$ at room temperature. The phonon wavevector modulus, $q$, is expressed in units of $2 \pi / a$, where " $a$ " is the lattice parameter of crystalline $\mathrm{Si}$, and " $\phi$ " is the diameter of the confinement volume expressed in units of the lattice parameter. One assumes cylindrical symmetry, with diameter much smaller than length according to the external aspect of the wires. The phonon dispersion relation for pure Si can be approximated by the following expression:

$$
\omega(q, T)=\left(A+\left\{A^{2}-B[1-\cos (\pi q)]\right\}^{1 / 2}\right)^{1 / 2}
$$

where $A(T)$ and $B(T)$ are determined from the phonon dispersion curve [7]. The Raman phonon bands depend on the temperature because of the thermal expansion and the anharmonicity of the vibrational potential energy $[5,8]$. The dependence of the Raman spectrum with temperature for bulk material was previously obtained on the control $\mathrm{Si}$ 
wafer. The variation of $\omega$ and $\Gamma_{o}$ with temperature were approached to a linear function:

$$
\begin{gathered}
\omega\left(\mathrm{cm}^{-1}\right)=521.9-0.0242 T\left({ }^{\circ} \mathrm{C}\right) \\
\Gamma_{o}\left(\mathrm{~cm}^{-1}\right)=2.8+0.0102 T\left({ }^{\circ} \mathrm{C}\right)
\end{gathered}
$$

The temperature coefficients of Eq. (3) for phonons with $q \neq 0$ are not available, therefore the full phonon branch was taken rigid and assumed to follow Eq. (3). This is only a first approximation, because one can expect a variation of the anharmonic constants of nanostructures with respect to the anharmonic constants of the bulk material [9].

Using this model we have estimated the effect of the temperature in the Raman spectra of the Si NWs [10]. The Raman band shifts to lower wavenumbers and broadens with the increase of temperature, becoming more symmetric respect to the Raman lineshape due to pure phonon confinement. For diameters higher than $20 \mu \mathrm{m}$, the effect of the phonon confinement is negligible, but the effect of the temperature increase is very important, shaping the one phonon band. By means of this model, we tried to adjust the experimental spectrum of Fig. 1, considering a unique diameter of the NWs, which is the fitting parameter $(\phi)$ in Eq. (1), failing in obtaining a reasonable good fit. The discrepancies arise from the non homogeneous temperature inside the scattering volume, which should be due to the inhomogeneous diameter distribution inside the probed volume, and the different incidence of the laser beam on the different NWs. In fact, as shown in the inset of Fig. 1, only a few NWs are probed in a Raman measurement, and the diameter and position of the laser beam with respect to them can change from each other.

In order to study the temperature distribution inside the NWs, we carried out finite element analysis of the heat dissipation in the NWs under the exposure to a laser beam with a Gaussian power profile. The calculation took account of the dependence of the thermal conductivity with the NWs diameter [11]. Two factors should be considered, the thermal dissipation, and the laser power energy absorbed. Concerning the absorbed energy, it should be considered the laser power density and wavelength; the heat dissipation is determined by the thermal conductivity, which depends on the diameter of the NWs and the surrounding media. For these calculations we considered a laser spot impinging in the centre of the NWs, with a power density of $100 \mathrm{kWcm}^{-2}$. The NWs are surrounded by air, which has a quite poor thermal conductivity $(0.024$ $\mathrm{W} / \mathrm{mK}$ ), the NWs being linked to the substrate by only one of the ends. The results of the simulations are summarized in Fig. 2.

The results demonstrated that the temperature inside the region of the NWs exposed to the laser beam presents a strong dependence with the NW diameter; therefore, significant temperature differences can be reached in between NWs of different diameter under the laser beam excitation. On the other hand, the axial temperature variation inside the laser beam in a single NW does not exceed $20{ }^{\circ} \mathrm{C}$ (not shown here). The effect of the length of the NWs is clearly visible, this effect being due to the fact that the NW can hardly dissipated heat towards the surrounding media (air), and only the base of the NWs, linked to the substrate, dissipates heat in an efficient way. This explains the thermal asymmetry observed in the NWs.

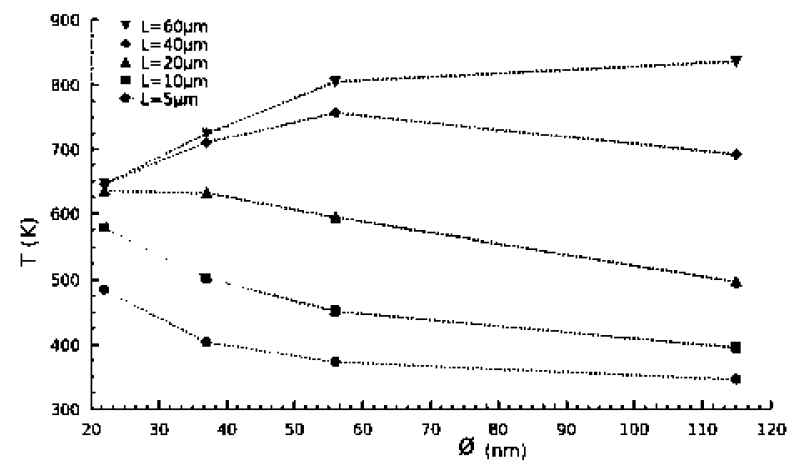

Figure 2 Calculated temperature induced by the laser beam as a function of the NW diameter $(\phi)$ for NWs with different lengths $(L)$ (incident laser power density $=100 \mathrm{kWcm}^{-2}$ ).

These data provided the information necessary to interpret the Raman spectra of discrete sets of Si NWs with dispersed diameters, which reached different temperatures. In particular, the Raman spectrum shown in Fig. 1 has been adjusted considering four NWs with diameters of 20,30 , 40 and $100 \mu \mathrm{m}$ (see the inset in Fig. 1), which according to our calculations reach temperatures of $400,325,250$ and $150^{\circ} \mathrm{C}$, respectively, obtaining a reasonable fitting of the experimental data [12].

The Raman spectra of individual $\mathrm{Si}_{\mathrm{x}} \mathrm{Ge}_{1-\mathrm{x}} \mathrm{NW}$ have been also obtained once the NWs have been isolated, Fig. $3(\lambda=633 \mathrm{~nm})$. In this case, the effect of the composition in the Raman spectra has to be also considered. The Raman spectrum was acquired crossing laterally an individual NW with an apparent diameter of $200 \mathrm{~nm}$, with a step size of $250 \mathrm{~nm}$ (see the inset of Fig. 3). These spectra are characterized by a broad peak consisting of two components, corresponding to the Ge-Ge vibrations, the broad band related to Si-Ge vibrations, also evidencing two contributions, and a band corresponding to $\mathrm{Si}-\mathrm{Si}$ vibrations, which only presents two components in selected zones. The splitting of the bands is due to the inhomogeneous composition of the NWs, because of the Ge segregation due to wire oxidation.

The Ge composition has been deduced from the intensity ratio $I_{\mathrm{Ge}-\mathrm{Ge}} / I_{\mathrm{Si}-\mathrm{Si}}$. We have tested this relation with a batch of epitaxial SiGe layers of known compositions, obtaining very good agreement. The peak positions, also related to the Ge composition [13] (assuming that the NWs are relaxed), was not used for this purpose, since they are expected to be affected by the laser heating, as demonstrated for Si NWs. In order to give a plausible explanation of this effect in this study, we also performed finite element analysis of the temperature increase for Si NWs in 
the case in which the laser spot impinges the sample offaxis. In this case, the distribution of the intensity of the laser spot in the NW is a crucial factor in determining the laser induced heating.

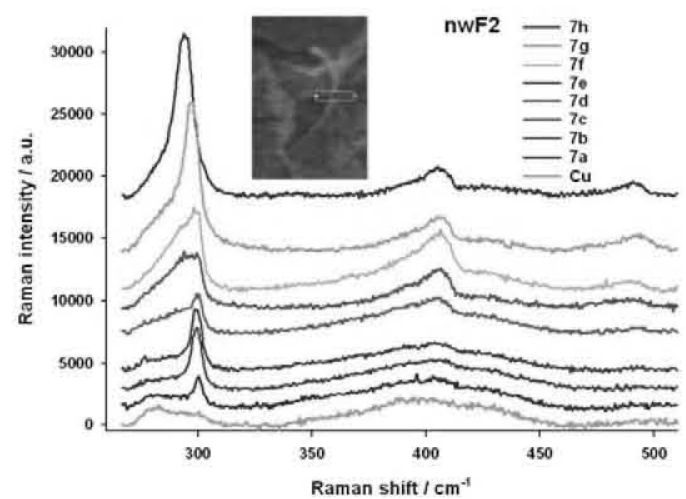

Figure 3 Raman spectra obtained by crossing an isolated SiGe NW (as shown in the inset).

Figure 4 shows the results of the maximum temperature reached by a Si NW of $6 \mu \mathrm{m}$ in length and $115 \mathrm{~nm}$ in diameter when the laser spot transversally cross the NW. In this case, following the experimental set-up, the NW has been considered on top of a holder, where only a line of the cylinder of the NW is in contact with the holder; thus, we assume that the heat dissipation is nearly negligible. In order to elucidate the effect of the position of the spot respect to the ends of the NW, the incidence point has been situated at two positions, corresponding to the middle of the NW (A) and close to the one of the ends (B) (at $1 \mu \mathrm{m}$ ). It is observed (Fig. 4) that there are nearly no differences between these two positions, which is due to the short length of the NW in this case. The differences are higher in the case of longer NWs. On the other hand, a very important temperature difference $\left(440^{\circ} \mathrm{C}\right)$ is observed between the situation in which the laser spot impinges only laterally the NW and the plain incidence in the middle of the NW. These results allow understanding the shift of the Raman bands observed in Fig. 3 as a consequence on the laser heating of the NW, depending on the position of the laser spot axis with respect to the NW axis.

4 Conclusions In this work we study, both experimental and theoretically, the Raman spectra of $\mathrm{Si}$ and $\mathrm{Si}_{x} \mathrm{Ge}_{1-x}$ NWs taking account of the excitation conditions and the surrounding media. Heating induced by the laser beam contributes in a critical way to shape the one phonon band, due to the low thermal conductivity of the surrounding media. A careful analysis of the experimental conditions, the dimension of the NWs, and the phonon band profiles is necessary to extract straightforward information of the Raman spectra of these structures.

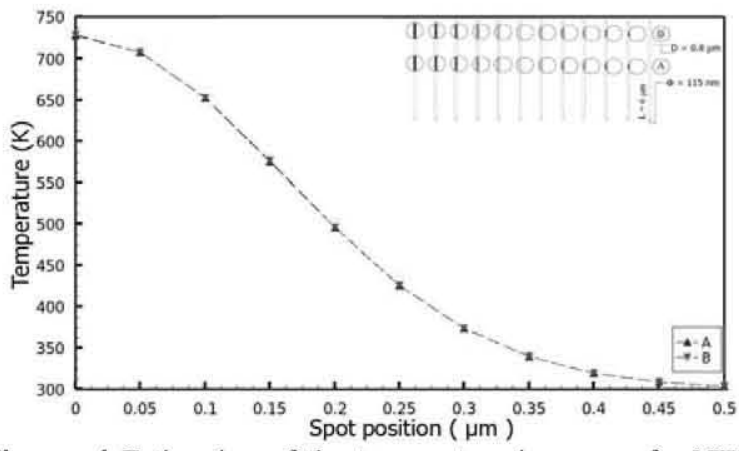

Figura 4 Estimation of the temperature increase of a NW as a function of the laser spot position ( $D=$ diameter of the laser spot) for a $\operatorname{SiNW}(L=6 \mu \mathrm{m}, \phi=115 \mathrm{~nm})$.

Acknowledgements This work was funded by the Spanish Government (Grant No. MAT-2007-66181) and by Junta de Castilla y León (Grant No. VA051A06 -GR202).

\section{References}

[1] Y. Cui and C. Lieber, Science 291, 851 (2001).

[2] S. Bhattacharyya and S. Samui, Appl. Phys. Lett. 84, 1564 (2004).

[3] R. Gupta, Q. Xiong, C. K. Adu, U. J. Kim, and P. C. Eklund, Nano Lett. 3, 627 (2003).

[4] H. Scheel, S. Reich, A. C. Ferrari, M. Cantoro, A. Colli, and C. Thomsen, Appl. Phys. Lett. 88, 233114 (2006).

[5] J. Jimenez, I. de Wolf, and J. P. Landesman, in: Microprobe Characterization of Semiconductors; Vol. 17, Optoelectronic Properties of Semiconductors and Superlattices, ed. by J. Jimenez (Taylor \& Francis, New York, 2002), ch. 2.

[6] I. H. Campbell and P. M. Fauchet, Solid State Commun. 58, 739 (1986).

[7] S. Wei and M. Y. Chou, Phys. Rev. B 50, 2221 (1994).

[8] J. Jimenez, A. Torres, E. Martin, and J. P. Landesman, Phys. Rev. B 58, 10463 (1998).

[9] M. J. Konstantinovic, S. Bersier, X. Wang, M. Hayne, P. Lievens, R. E. Silverans, and V. V. Moshchalkov, Phys. Rev. B 66, 161311(R) (2002).

[10] A. Torres, O. Martinez, C. Prieto, J. Jimenez, A. Rodriguez, J. Sangrador, T. Rodriguez, Mater. Res. Soc. Symp. Proc. 1145, 1145-MM04-12 (2009).

[11] D. Li, Y. Wu, P. Kim, L. Shi, P. Yang, and A. Majumdar, Appl. Phys. Lett. 83, 2934 (2003).

[12] A. Torres, A. Martín-Martín, O. Martínez, A. C. Prieto, V. Hortelano, J. Jiménez, A. Rodríguez, J. Sangrador, and T. Rodríguez, Appl. Phys. Lett. 96, 011904 (2010).

[13] F. Pezzolli, L. Martinelli, E. Grilli, M. Guzzi, S. Sanguinetti, M. Bollani, H. D. Chrastina, G. Isella, H. von Kanel, E. Winsterberger, J. Stangl, and G. Bauer; Mater. Sci. Eng. B 124, $127(2005)$. 